\title{
Chlorhexidine - An antiseptic in periodontics
}

\author{
Dr.Poonam Dutt ${ }^{1}$, Dr. Promod Kr Rathore ${ }^{2}$, Dr. Dheeraj Khurana ${ }^{3}$ \\ I'Professor, Dept. of Periodontics, Shree Bankey Bihari Dental college, Ghaziabad, U.P., India) \\ ${ }^{2}$ (Senior Lecturer, Dept. of Periodontics, Shree Bankey Bihari Dental college, Ghaziabad, U.P., India) \\ ${ }^{3}$ (P.G Student, Dept. of Periodontics, Shree Bankey Bihari Dental college, Ghaziabad, U.P., India)
}

\begin{abstract}
Studies have demonstrated that plaque microorganisms are the primary etiologic factors associated with human periodontal disease, plaque must be removed on a consistent basis as a preventative measure. Unfortunately, routine use of oral hygiene aids (e.g. toothbrush, floss, etc.) to control plaque have not been entirely successful. Accordingly, the search for alternative methods, such as chemotherapeutic agents, to control plaque has been extensive. Of the many chemical agents that have been evaluated to determine their effects on plaque, gingivitis and adult periodontitis, Chlorhexidine (CHX) have been successfully introduced and extensively researched. Chlorhexidine have been used to aid in controlling plaque build-up. They have been used to complement, not replace mechanical therapy.
\end{abstract}

Keywords: Antiseptic, Chlorhexidine, Dental Plaque, Periodontitis, Tooth Discolouration.

\section{Introduction}

As an antimicrobrial agent, chlorhexidine is effective in vitro against both Gram-positive and Gramnegative bacteria including aerobes and anaerobes and yeasts and fungi. The digluconate of chlorhexidine (1:6Di 4'-chlorophenyl-diguani-dohexane) is a synthetic antimicrobial drug which has been widely used as a broad spectrum antiseptic in clinical and veterinary medicine since 1953(1-3).

They are agents that kill oral microorganisms that cause gingivitis, periodontitis, and caries(4). Its antibacterial action is due to an increase in cellular membrane permeability followed by coagulation of the cytoplasmic macromolecules(5). It has also been shown that chlorhexidine can reduce the adherence of Porphyromonasgingivalis to epithelial cells(6). This effect is probably due to the binding of chlorhexidine to the bacterial outer membrane and therefore it could have similar results on the adherence of other plaque bacteria(7).

The most prevalent infectious oral diseases in humans, caries and periodontal diseases, are associated with dental plaque. The removal of bacterial biofilm is a decisive component in the prevention and treatment of these diseases. Because of the difficulty to ensure adequate removal of plaque by mechanical means, there is a great interest in the use of antimicrobial agents to replace or to be adjuncts to the mechanical approaches. Chlorhexidine (CHX) is one of the most effective antimicrobial agents for plaque control. It is retained in the oral cavity and is progressively desorbed in bacteriostatic concentrations 8 hours after rinsing. When a low dose of CHX is used, the cellular trans- port of the bacterial cell is damaged with the creation of pores in the cellular membrane. In higher concentration, the solution penetrates the bacterial cell and leads to microorganism destruction(8).

\section{Structure}

Chlorhexidine (CHX) is a bisbiguanide antiseptic that is a symmetrical molecule consisting of four chlorophenyl rings and two biguanide groups connected by a central hexamethylene bridge. The compound is a strong base and is di- cationic at $\mathrm{pH}$ levels $>3.5$, with the two positive charges on either side of the hexamethylenebridge(9). Several bisguanide antiseptics possess anti-plaque activity, including chlorhexidine, alexidine andoctenidine. Chlorhexidine gluconate, however, is the most studied bisguanide and is the one on which there is most information on toxicology. It acts by altering integrity of cell membrane of bacteria. Its superior anti-plaque activity is the result of its substantivity and pin-cushion effect(10).

\section{Substantivity Of Chlorhexidine}

The ability of drugs to adsorb onto and bind to soft and hard tissues is known as substantivityand this property was first described for chlorhexidine in the 1970s. Substantivity is influenced by the concentration of the medication, its $\mathrm{pH}$ and temperature, and the length of time of contact of the solution with the oral structures. This property of chlorhexidine was associated with its ability to maintain effective concentrations for prolonged periods of time(11) and this prolongation of its action made it especially suitable for the inhibition of plaque formation(12). 


\section{Pin-Cushion Effect}

One charged end of Chlorhexidine molecule binding to the tooth surface and the other remaining available to interact with bacterial membrane as microorganism approaches the tooth surface a pin cushion effect. This explains the lack of effectiveness of other antimicrobials in terms of them lacking a large, rigid molecule with two charged interactive ends(13).

\section{Metabolism And Toxicity}

When Chlorhexidine is used as a mouth rinse the mode of action is purely topical. The drug does not penetrate oral epithelium and if some solution is inadvertently swallowed, initial binding of the drug will be to the mucosal surfaces of gastrointestinal tract. Chlorhexidine is poorly absorbed through gastrointestinal tract. Animal experiments have suggested that Chlorhexidine is mainly excreted through feces. The small amount of Chlorhexidine that may be absorbed is metabolized in liver and kidney. There is minimal metabolic cleavage. It is free from systemic toxicity in oral use \& microbial resistance \& superinfection do not occur. The oral LD 50 value for Chlorhexidine digluconate is $1800 \mathrm{mg} / \mathrm{kg}$, whereas the LD 50 for intravenous application is $22 \mathrm{mg} / \mathrm{kg}(14)$.

\section{Availability}

Over the years, CHX in gels, chips, sprays, and mouth- washes hasbeendeveloped.Ofthese,amouthwash is the most commonly used(15).

\section{Use As A Mouthwash}

At present, there are 2 commonly used CHX concentration available: one, $0.2 \%$ CHX, which is used with a $10 \mathrm{ml}$ volume and the other $0.12 \% \mathrm{CHX}$, which is used with a $15 \mathrm{ml}$ volume. The rationale for lowering the concentration of CHX is to reduce side effect while maintaining comparable efficacy. Although the concentration in these 2 products are different, the total amount of CHX is approximately the same: $10 \mathrm{ml}$ of $0.2 \%$ CHX contains $20 \mathrm{mg}$ and $15 \mathrm{ml}$ of $0.12 \%$ CHX contain $18 \mathrm{mg}$ per volume. Although CHX is an effective antimicrobial agent in both concentrations(16).

When prescribed, it is recommended that patients rinse twice a day for 30 seconds with $15 \mathrm{~mL}$ after brushing and flossing and after toothpaste has been completely rinsed out of the mouth(4). Chlorhexidine is recommended as a twice daily, morning and evening, oral rinse to be used for at least 30 seconds. It is not intended for ingestion and should be expectorated after rinsing(17).

\section{Use As A Toothpaste}

More recently, some toothpastes have been specifically formulated to ensure a high availability of the contained antiseptic. A 1\% chlorhexidine toothpaste of this type has been investigated in a 19- day, randomized double blind, placebo-controlled, cross-over experimental gingivitis clinical trial. The toothpastes were used as slurries which were rinsed around the mouth twice per day for 1 minute during the experimental period. Plaque and gingivitis scores were highly significantly reduced and stain scores were significantly increased in the active toothpaste period with respect to those in the placebo period. Thus, this particular formulation of chlorhexidine toothpaste does seem to provide a sufficient dose of chlorhexidine for a similar clinical effect to that seen with chlorhexidine mouth rinsing(18).

\section{Use As Gum}

Chlorhexidine has also been incorporated into a sugar-free chewing gum (Fertin A/S, Vejle, Denmark), and in this form the chlorhexidine molecule remains unbound. The chewing gum contains $20 \mathrm{mg}$ of chlorhexidine diacetate and this has been compared with the effects of a $0.2 \%$ chlorhexidine mouthwash and a placebo gum in a clinical study. Therefore, the use of chlorhexidine gum could be a good method of using chlorhexidine in longer term users(18).

\section{Use As Locally Applied Antiseptic}

Periochip (Dexcel Pharmaceuticals, Israel) is an orange-brown, biodegradable, rectangular chip rounded at one end that has an active ingredient of chlorhexidine gluconate $(2.5 \mathrm{mg})$ that is released into the pocket over a period of 7 to 10 days. It has been found to suppress the pocket flora for up to 11 weeks post application(4).

Periochip is the only locally applied non-antibiotic antimicrobial approved by the FDA as an adjunct to SRP procedures for the reduction of probing pocket depth or as part of a routine periodontal maintenance program. The recommendation for use adjunctive to SRP involves isolation of the periodontal pocket of $5 \mathrm{~mm}$ or more, drying the surrounding area, and grasping the Periochip with a forceps and inserting the chip, curved 
end first, into the pocket to its maximum depth. The chip can be maneuvered further into position with a plastic instrument. One site can be treated per chip(4).

\section{Use As Spray}

Sprays could well be used as a replacement for, or an adjunct to, mechanical tooth-brushing. This is especially true in situations in which mechanical tooth cleaning is impossible and the use of a mouthwash is difficult or not possible at all. Therefore, indications for the use of CHX spray could include mentally handicapped patients, elderly persons in nursing homes, patients in intensive care units in hospitals, and patients with inter-maxillary fixation. Sprays have the advantage of focusing delivery at the required site. Therefore, spray also was advocated for local application in cases of implantology and periodontal surgery(15).

The efficacy of sprays depends on the operator, who should achieve coverage at all sites, as well as the total dose that is delivered. In this respect, itis interestingtonote that no difference in efficacy was found between $0.2 \%$ CHX spray and $0.2 \%$ CHX mouthwash. Also, no difference was found between $0.2 \%$ spray and $0.12 \%$ mouthwash. No data are available on the comparison of $0.2 \%$ and $0.12 \%$ sprays in terms of plaque growth inhibition(15).

\section{Use As Gel}

Topical application of Xanthano GEL (CHLO-SITE) which contains a mixture of chlorhexidine digluconate and dihydrochloride, in a ratio of 1:2.Local administration of chlorhexidine has been demonstrated to be effective as periodontal therapy. Its mechanism of action includes reduction pellicle formation, alteration of bacterial adherence to teeth, and alteration of bacterial cell walls which causes cell lysis(19).

\section{Side Effects}

Chlorhexidine is poorly absorbed by the gastrointestinal tract and it therefore dis- plays very low toxicity (oral LD50 is $1800 \mathrm{mg} / \mathrm{kg}$ and the intra- venous LD50 is $22 \mathrm{mg} / \mathrm{kg}$ ). No tetragenic alterations have been found following long-term use(18).

The reported side effects of $\mathrm{CXH}$ are alteration in taste, increase of calculus formation, staining of teeth and mucous membranes and, more rarely, oral mucosa desquamation and parotid swelling. However, the most obvious and important local side effects are the brown staining of the teeth, restorative materials and dorsum of the tongue as well as supragingival calculus formation.

Non-enzymatic browning (Maillard reactions) and formation of pigmented metal sulfides are considered the possible mechanisms of tooth discolorations due to CHX application. However, clinical and laboratory studies provided strong evidence that staining is caused by interaction or precipitation of dietary chromogens with locally adsorbed $\mathrm{CXH}$. In the presence of food components, CHX dyes produce colored compounds on hydroxyapatite. Moreover, when associated with tea and coffee intake, brown staining of tooth and acrylic previously exposed to CXH is more likely to occur. Corroborating these findings, Addyet. al. (1979) demonstrated that tea, coffee and red wine were particularly chromogenic(20).

The increase of calculus formation due to CHX mouthrinse is a usual finding in early long-term investigations. However, short-term studies suggested reduced calculus formation with CHX rinsing.The process of mineralization and the CHX influence on the process are not fully understood, but involves localized supersaturation, nucleation, crystal growth and the transformation of precursor phases such as dicalcium phosphate dehydrate, octacalcium phosphate and amorphous calcium phosphate into more stable, crystalline deposits of hydroxyapatite.However, a recent in vivo study has shown that adjunctive usage of an oxidizing agent in addition to chlorhexidine reduced such staining and plaque formation and development of gingivitis(20).

Lesscommonly,CHXcausesmucosalerosion, and this appears idiosyncratic, with the antiseptic effects on the surface of epithelial cells in some people. Desquamative soft tissue lesions have also been reported with use of drug concentrations exceeding $0.2 \%$ or after prolonged application. Extremely rare but unilateral or bilateral parotid swelling may occur following use of CHX rinsing, but the mechanism is unknown(17).

For these reasons, the prolonged use of chlorhexidine should be avoided in normal periodontal patients. It is useful for short periods of up to 2 weeks when oral hygiene may be difficult or impossible, such as during acute oral infections or following periodontal or other forms of oral surgery. It may occasionally be used as an adjunct to mechanical oral hygiene in initial periodontal treatment. In this regard, the gingivae may be sore after subgingival scaling and this may prevent normal tooth brushing. However, this effect does not usually last more than 2 to 3 days and therefore the use of the mouthwash is not usually necessary for more than this period. At this point normal brushing and flossing must be resumed and mouthwashing should stop(18). 


\section{Conclusion}

Chlorhexidine to date is the most potent anti plaque agent. Chlorhexidine and any other potent antimicrobial agent should be applied only under professional supervision.

\section{References}

[1]. G. Davies, J. Francis, A. Martin, F. Rose, G. Swain, 1:6 Di-4'-chlorophenldiguanidohexane. Laboratory investigation into a new antibacterial agent of high potency, Br J Pharmacol, 9, 1954, 192-196.

[2]. T. Hennessy, Some antibacterial properties of chlorhexidine, J Periodont Res, 8(suppl.), 1973, 61-67.

[3]. C. Emisilon, Susceptibility of various microorganisms to chlorhexidine. Scand J Dent Res, 85, 1977, $255-265$.

[4]. ME. Ryan, Nonsurgical approaches for the treatment of periodontal diseases, Dent Clin N Am, 49, 2005, 611-636.

[5]. T. Hennessy, Antibacterial properties of Hibitane, J ClinPeriodontol, 4, 1977,36-48.

[6]. D. Grenier, Effect of chlorhexidine on the adherence properties of Porphyromonasgingivalis, J ClinPeriodontol, $23,1996,140-142$.

[7]. P. Hull, Chemical inhibition of plaque, J ClinPeriodontol, 7, 1980, 431-442.

[8]. CAF. Neto, CCF. Parolo, CK. Rösing, M. Maltz, Comparative analysis of the effect of two chlorhexidine mouthrinses on plaque accumulation and gingival bleeding, Braz Oral Res 22(2), 2008, 139-44.

[9]. L. Flo"tra,P. Gjermo, G. Rolla, J. Waerhaug, Side effects of chlorhexidine mouthwashes, Scand J Dent Res, 79, 1971, 119-125.

[10]. M. Addy, Chlorhexidine compared with other locally delivered antimicrobials. J ClinPeriodontol, 13,1986, 957-964.

[11]. P. Bonesvoll , P. Lökken, G. Rölla, Influence of concentration, time, temperature and pH on the retention of chlorhexidine in the human oral cavity after mouth rinses. Arch Oral Biol, 19, 1974, 1025-1029.

[12]. Winrow M. Metabolic studies with radiolabelled chlorhexidine in animals and man. J Periodont Res, 12, (suppl.), 1973, 45-48.

[13]. S. Mathur, T. Mathur, R. Srivastava, R. Khatri, Chlorhexidine: The Gold Standard in Chemical Plaque Control, National Journal of Physiology, Pharmacy \& Pharmacology, 1(2), 2011, 45-51.

[14]. E. Faulkers, Some toxicological observations on Chlorhexidine, J. Periodontol Res, 8 (suppl 12), 1973, 55-57.

[15]. JE. Stoeken, PA. Versteeg, NAM. Rosema, MF. Timmerman, U. van der Velden, GA. van der Weijden, Formation With 0.12\% Chlorhexidine Spray Compared to 0.2\% Spray and 0.2\% Chlorhexidine Mouthwash, J Periodontol, 78, 2007, 899-904.

[16]. JAM. Keijser, H. Verkade, MF. Timmerman, FA. Van der Weijden, Comparison of 2 Commercially Available Chlorhexidine Mouthrinses, J Periodontol, 74, 2003, 214-218.

[17]. CA. Gürgan, E. Zaim, I. Bakirsoy, E. Soykan, Short-Term Side Effects of 0.2\% Alcohol-Free Chlorhexidine Mouthrinse Used as an Adjunct to Non-Surgical Periodontal Treatment: A Double-Blind Clinical Study, J Periodontol, 77, 2006, $370-384$.

[18]. BM. Eley, Antibacterial agents in the control of supragingival plaque - a review, British Dent. journal, 186(6), 1999, 286-296.

[19]. MMAJ. Abdaly, ANMEl. Refai, UM. Gouda, HA. Atty, Evaluation of Topical Application of CHLO-SITE (Chlorhexidine gel) in Management of Chronic Periodontitis, Suez Canal Univ Med J, 11(1), 2008, 35 -40.

[20]. FB. Zanatta, RP. Antoniazzi, CK. Rösing, Staining and calculus formation after $0.12 \%$ chlorhexidine rinses in plaque-free and plaque covered surfaces: a randomized trial, J Appl Oral Sci, 2009, 515-521. 